\title{
ANALYSIS OF HIGH FRAME-RATE MOVIES BY VARIATIONAL METHODS
}

\author{
MASSIMILIANO PEDONE, SILVIA CAROSIO, GIANCARLO RUOCCO \\ AND ZACCARIA DEL PRETE
}

\begin{abstract}
The object of this study was the recognition of Regions Of Interest(ROIs) in a time series of digital images of two specific laboratory experiments. It concerns the identification of objects in a tissue surface by high-resolution and high-speed ad-hoc systems for morphological dynamic image analysis. The protocols and the algorithms implemented are developed to retrieve biomechanical properties of two different in vitro systems; the solid filament X-MET (eX-vivo Muscle Engineered Tissue) to measure its reaction to a different frequency stimulation, and a planar system of co-culture of skeletal and cardiac muscle cells, where myotubes and cardiomyocytes coexist, to discriminate the interaction between different cell's type, of its spontaneous pulse. The results of the stimulated X-MET from solid culture system are frequency dependent points of the macroscopic muscular strength and its contractile response. The results for the co-culture planar board measure the correlation of the pulsed movements of the different parts of the tissue.
\end{abstract}

\section{INTRODUCTION}

At the IIT@CLNS Biomechanics Laboratory of Rome the researchers have tested a system based on Digital Image Correlation (DIC) [11] for the measurement of small high dynamic biological muscle specimens' contractile properties, that in some cases fails, especially on X-MET muscle tissues movements. Our goal, in this field of investigation, is to build ad-hoc reliable methods to track objects during their movements. We focus on two different in vitro systems of muscle cells analysis: one is filament of muscle fiber and the other is planar culture, both processing algorithms implemented by Mumford Shah energy functional minimization [1] in 2 and 3 dimension. The X-MET muscle filament, is electrically stimulated, and we need to retrieve its strength reaction measure. The planar in vitro co-culture system presents myotubes and cardiomyocytes in spontaneous contraction. Once the skeletal muscle cells have formed multinucleated myotubes ( 5 days after plating), myotubes show spontaneous contractile activity without any stimulation, while, the day after isolation, cardyomyocites shows spontaneous contraction $[5,9]$.

To monitor the contraction and the possible electrical coupling of skeletal and cardiac cells, an experimental system is in course of development. We perform an analysis of pair of selected ROIs, to establish the interaction and correlation of these contractile movements.

$M S C$ (2010): primary 49-04, 68U10; secondary $65 \mathrm{~K} 15$.

Keywords: variational methods, muscle engineered tissue, image and movie processing. 


\subsection{The X-MET system}

The analysis of the X-MET contraction is based on a high resolution microscope joint with a high efficiency camera [11], able to record up to 400 frame per second of $1020 \times 300$ pixels gray scale images Fig. 1 . The post-processing analysis consists of two phases. We have to filter the single frame to enhance the edge of cell and obtain a regularized object.

Different kind of pre-processing techniques can be adopted and lots of regularization techniques can be tried, the previous study on the echocardiographic movies [8], suggests an approach based on the energy functional minimization; because it preserves the edge of the little areas, as the cells on the muscle surface, better than the smoothing-based methods. The objects visible in the tissue are difficult to track; sometimes the jerking movement is greater than the cell dimensions, faster than the frame-rate and vibration, on the vertical axis, cause focal distortions of microscope lens and the software DIC for tracking rigid causes errors and disconnects its follower algorithm.

We choose the dynamic regularization technique of Mumford-Shah in order to establish the parameter values $\lambda$ and $\alpha$ for functional minimization. The goal is to select an object on the muscle surface; enough smooth and with an alphaproportional length of the perimeter. This approach limits the tracking error or recovers it in a few steps, as we explain in the next section. The selected ROIs are mapped in a record structure containing its dimension, border, centroid relative and absolute position, label, and area.

To study the X-MET behavior, the synchronized measurement of strain and force makes it possible to obtain a comprehensive tool for tissue's monitoring. In the muscle filament system, one end of the X-MET is anchored while the other end is fixed to a micro force transducer, so we can retrieve an isometric measure of the strength during stimulation, see [10].

When electrically stimulated by means of two platinum electrodes, X-MET shows a contractile response that is consistent with that of adult skeletal muscles: single pulses evoke twitch contraction, while trains of pulses elicit tetanic contractions which produce forces that increase when the frequency increases [4]. We retrieve the results for different stimulation frequency and on every selected ROI. Thus we now present a numerical frame pre-processing technique based on a variational model that consists of functional minimization with a Mumford-Shah (M-S) time-dependent energy term, which is suitable to enhance ROI's edge and regularize initial data for the "follower" algorithm. Our goal is to regularize the frame to enhance, select, and follow the ROI's centroid, (see Fig. 2) frame by frame. The contribution is to build a protocol of elaboration that performs the recognition of the ROIs to evaluate the local strength of the muscle fiber against the force transducer global measurements. So we need to perform a great deal of manually driven adjustment of the parameter involved in the functional minimization. From a 3D point of view, the "follower" is developed to perform the continuous segmentation of the region, "like a hole in cheese", and evaluate at every step the region properties. 


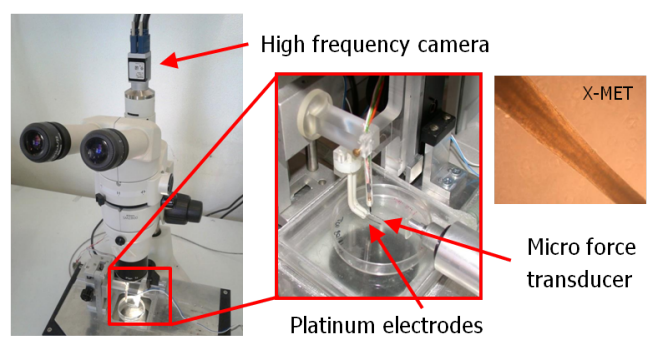

Figure 1. The X-MET system, the microscope (stereomicroscope NIKON SMZ 800 for tissue magnification up to $6.3 \mathrm{X}$ ) over the sample plate with high frame rate camera (Vendor Basler Model acA2040-180km, Type of data presented Selected samples, Sensor type CMOS, Sensor diagonal Diagonal $15 \mathrm{~mm}$, Resolution 2048×2048 pixel, Pixel width $5.50 \mu \mathrm{m}$, Pixel height 5.50 $\mu \mathrm{m}$ ), the muscle filament are in the middle of two platinum electrodes that provide the electrical stimulus by a electrostimulator. One end of the muscle is anchored to a piezoelectric micro force transducer to measure the strength [11].

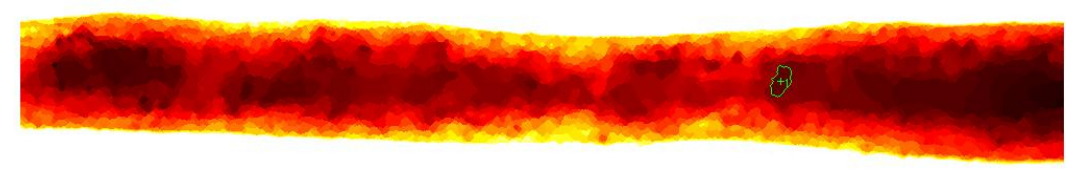

Figure 2. Selected ROI 01 (green contour and + the centroid position) over the real frame $(1005 \times 140$ pixel $)$ in hot color map, for a better identification of the different zone where the muscle strength can be measured.

\subsection{The co-culture system}

The planar cell co-cultures were obtained seeding skeletal muscle cells onto 15and-half-day old embryonic cardiomyocytes. To enhance cardiomyocyte survival and the correct adhesion to the culture dish, it was necessary to leave the cells in their specific culture medium for 48 hours. After this period, skeletal muscle cells, obtained from a primary culture, were seeded on cardiac cells and cultured in skeletal muscle growth medium [4]. The planar in-vitro co-culture model exhibits cell spontaneous activity: the contraction of differentiated myotubes and the beating of cardiomyocytes. To evaluate the possible electrical coupling of skeletal and cardiac muscle cells, we have to build an ad-hoc method of analysis. The previous applications by Mumford Shah, on echographics images [8] and the tests with the planar model don't give enough edge continuity over the frames. The low frame rate causes a gear deal of edge presence in every frame not enough continuous. So the idea was to see the time dimension as the third dimension of the domain set in functional minimization. Let $\Omega$ the image domain and $g, u, S \in \Omega \subset \mathbb{R}^{2} \times[0, \ldots, T] \rightarrow \mathbb{R}$. In Fig. 3 we distinguish the circular cardiomyocytes and skeletal muscle myotubes of long profile. The low frame rate, about 15 fps, cannot give a real continuous edge detection in $2 \mathrm{D}$ and the follower cannot recognize the motion of a single ROI. By a $3 \mathrm{D}$ pre-processing technique, we obtain regularized areas, over the frames, representing different ROIs. Thus, we can adopt the frame to frame edge selection on regularized image by a manual driven 


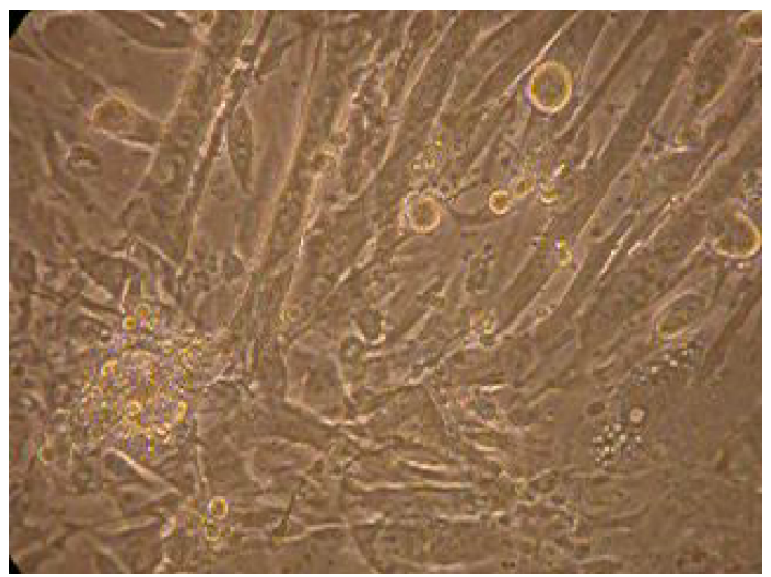

Figure 3. Co-culture system, circlular cells identify cardiomyocytes and long aggregates was myotubes. Their rhythmical pulse seems to be uncoupled but correlated.

threshold filtering. The modified M-S for a 3D domain, by a fine screw-driver adjustment of the parameter; $\lambda, \alpha$, give us good results and the pre-processed $3 \mathrm{D}$ array can be passed to the Matlab $(\mathrm{R})$ "follower" algorithm described in the next part.

\section{Model PROBLEM}

We search for constituent parts of an image. Our aim is to enhance the edges of the various cells captured in the frames by the acquisition system. We enhance and detect the objects visible on the muscle fiber by functional minimization. We assume these objects to be Regions of Interest and follow their rhythmical movements. We use the Mumford-Shah (M-S) functional [7] in its approximated form proposed by Ambrosio Tortorelli [1] to segment the cell borders. An accurate choice of the parameter $\lambda, \alpha \in \mathbb{R}^{+}$that perform the best recognition of the ROIs is needed for each particular movie. In the following section, we briefly recall the essential formulation of the model problem used to regularize and enhance images. For a complete review, see the book by Morel and Solimini [6].

The variational model introduced by Mumford and Shah in 1985, consists of an energy-based method to minimize over the pair $u$ and $\Sigma$ the functional

$$
\int_{\Omega}(u-g)^{2}+\lambda \int_{\Omega \backslash \Sigma}|\nabla u|^{2} d x+\alpha \mathcal{H}^{d-1}(\Sigma),
$$

in the domain $\Omega \subset \mathbb{R}^{d}$. The function $g: \Omega \rightarrow[0,1]$ represents a gray-scale image encoding, at each point of the bounded open subset $\Omega \subset \mathbb{R}^{d}$ domain , our image in the explained application for $d=2,3$ (planar or solid approach). For a given image $g \in L^{\infty}(\Omega)$ we search for a function $u: \Omega \subset \mathbb{R}^{d} \longrightarrow \mathbb{R}$ and $\Sigma$ is a finite Hausdorff (d-1)-dimensional measure $\mathcal{H}^{d-1}(\Sigma)$ closed set and represents the set where the $u$ can be discontinuous. The set $\Sigma$ consists of the edges of the segmented objects contained in the given image $g . \Sigma \subset \Omega$ (the discontinuity set) Mumford and 
Shah conjectured that the functional admits a minimizer satisfying (C1) and (C2) regularity hypotheses [7].

(C1) $\Sigma$ consists of a finite number of $C^{1,1}$-curves, meeting $\partial \Omega$ and meeting each other only at the endpoints.

(C2) $u$ is $C^{1}$ on each connected component of $\Omega-\Sigma$.

The minimization is a hard task mainly due to the term depending on the measure of the surface $\mathcal{H}^{d-1}\left(S_{u}\right)$. Several different approaches have been proposed in the last decade, we use the most popular one proposed by Ambrosio and Tortorelli [1].

\subsection{Ambrosio-Tortorelli approximation of the M-S Functional}

The bidimensional approximation of the M-S functional to search for a minimum by Euler equation has been presented so far. The numerical solution of the equation is just a guarantee for local minima, while $\Gamma$-convergence assures for the functional the existence of global minima under the regularity hypotheses, as we explain in the next section. We now consider the weak version of the functional; where $u$ is in the space SBV of the special function of bounded variation (having distributional gradient without Cantor part), let the functional:

$$
F_{M S}(u)=\int_{\Omega}(u-g)^{2} d x+\lambda \int_{\Omega}|\nabla u|^{2} d x+\alpha \mathcal{H}^{1}(S u),
$$

where the minimization is made only over the function $u$ and the role of $K$ is assumed by the $S u$ representing the unknown set of discontinuity point, from minimization theorem 3.1 Proposition 2.1 in [3], it respectively follows that:

$S_{u}$ is a piecewise $C^{2}$ submanifold of $\mathbb{R}^{2}$. Non-convexity of the functional is given by its geometric part. Nevertheless, parameters generate different weights for individual addenda; their role, for limit values, determine how much cost the jumps of the geometric part of the weak form. Let $\lambda=1$, we consider the role of the parameter $\alpha$ in the weak form of the functional:

$$
F_{M S}(u)=\int_{\Omega}(u-g)^{2} d x+\int_{\Omega}|\nabla u|^{2} d x+\alpha \mathcal{H}^{1}(S u) .
$$

When $\alpha \rightarrow+\infty$, the term $\alpha \mathcal{H}^{1}(S u)$ vanishes over the jump set. Consequently, the functional gets convex

$$
F_{M S}(u)_{\alpha=\infty}=\int_{\Omega}(u-g)^{2}+\int_{\Omega}|\nabla u|^{2} d x .
$$

By a continuation method, if $\alpha_{0}=10^{6}$ is taken, we obtain a convex functional again.

For the geometric part, very large parameters $\alpha$ and $S=1$ are typically used

$$
\alpha \int_{\Omega}\left(\epsilon|\nabla S|^{2}+\frac{1}{4 \epsilon}(1-S)^{2}\right) d x .
$$

The procedure consists of solving an Euler equation system for the $\alpha$-values which are progressively reduced, for example $\alpha_{k}=\alpha_{0} c^{k}$ with $0<c<1$. Thus, heuristically, one goes down along the functional gradient until the global minimum is reached, with a functional slightly deconvexed.

For different $\lambda>0$ and $\alpha>0$, the three addenda can be explained as follows: 
I) $L^{2}$-distance of $u$ from the given $g$, which evaluates how much our image solution is "near" to the given image.

II) To make small areas, delimited by small contours, not distinguishable, our $u$ must be $C^{1}$ in large subsets. The presence of the $\lambda$-parameter allows us to smooth the function $u$ as much as we like. Following the optical flow model (see page $184[2]$ ), we introduce the time dependence in the gradient term $\left(|\nabla u|^{2}\right)$ explained in the numerical section. The gradient of $u$ for the examined frame is calculated with its neighborhoods. We formulate the gradient calculus in the next part to explain the time dependence of its values for a three consecutive frames. So we increase the presence of the edges with a "continuous motion".

III) In this addendum we add a measure of segmentation spread by considering the length of curves. When the value of the parameter $\alpha$ is very small, small contours are privileged, while larger values privilege large subsets. Thus, the values of $\alpha$-parameters are usually chosen to allow for a correct recognition of forms according to the expectations and type of image. Many different choices of $\alpha$-parameter values have been made in our application for the recognition of the interested area.

We use the standard methods of Ambrosio-Tortorelli sequence of approximated functionals [12] to calculate the relative Euler equations. By the Birindelli, Finzi Vita [3] technique, we can divide the problem into the sequences of uncoupled elliptic system of equations [8].

\subsection{Sketch of $\Gamma$-convergence for the $\mathrm{M}-\mathrm{S}$ functional}

We recall the main property of the $\Gamma$-convergence of Functionals by the meaning convergence of minimizers up to a subsequence like the Ambrosio Tortorelli form of the M-S Functional for its equi-coercive property. The weak form $F(u)$ of the M-S Functional take its argument $u$ on the Special Bounded Variation space (SBV) for function without Cantor part in its distributional gradients [1,12]. The $\Gamma$-convergence for the weak form of the functional is obtained up to a subsequence of minimizers where the equi-coercivity is assured for the tubular neighborhood construction. Briefly, in Ambrosio Tortorelli's theorem the functional $F$ is approximated by a sequence of elliptic functionals. Let the pair $(u, \Sigma)$ the minimizers of the Functional $F(u)$ then the functional is defined by

$$
\widehat{F}(u, S)=\left\{\begin{array}{cc}
F(u) & \text { if } u \in S B V(\Omega), S \equiv 1, \\
+\infty & \text { otherwise. }
\end{array}\right.
$$

Where the set $\Sigma$ is replaced by an auxiliary variable $S$ (a function) which approximates the characteristic function $\left(1-\chi_{\Sigma}\right)$.

Let $\left\{K_{\epsilon}\right\}$ be a sequence of positive numbers converging to zero such that

$$
\lim _{\epsilon \rightarrow 0^{+}} \frac{K_{\epsilon}}{\epsilon}=0
$$

The approximating functional

$$
\widehat{F_{\epsilon}}(u, S)=\int_{\Omega}(u-g)^{2} d x+\lambda \int_{\Omega}\left(S^{2}+K_{\epsilon}\right)|\nabla u|^{2} d x+
$$




$$
+\alpha \int_{\Omega}\left(\epsilon|\nabla S|^{2}+\frac{1}{4 \epsilon}(1-S)^{2}\right) d x
$$

Where the above term $\left(S^{2}+K_{\epsilon}\right)$ realizes, when $\epsilon \rightarrow 0^{+}$, the shrinking tubular neighborhood. The approximation of the sequence of functional in M-S theory in the discontinuity set $[1,12]$ by the $\mathrm{A}-\mathrm{T}$ subsequence. For the equi-coecivity property of the approximating functional, there exists a convergent subsequence of minimizers [12] to the M-S solution.

\subsection{Ambrosio-Tortorelli approximation of the M-S functional}

We refer to algorithm for the approximation of $F(u, \Sigma)$ with a sequence $F_{\epsilon}$ of regular functionals defined on a Sobolev space. In image analysis the most used is the Ambrosio-Tortorelli approximation [2]. Let the parameter $\alpha=1$ and $\lambda=1$, we can write:

$F_{A T \epsilon}(u, S)=\int_{\Omega}(u-g)^{2} d x+\int_{\Omega} S^{2}|\nabla u|^{2} d x+\int_{\Omega}\left(\epsilon|\nabla S|^{2}+\frac{1}{4 \epsilon}(1-S)^{2}\right) d x$.

If $\left(u_{\epsilon}, S_{\epsilon}\right)$ minimizes the functional $F_{\epsilon}$, then the following result holds, see [1]:

$$
u_{\epsilon} \stackrel{\mathcal{L}^{2}}{\longrightarrow} u \text { ed } S_{\epsilon} \longrightarrow 1-\chi_{\Sigma} \text { per } \epsilon \longrightarrow 0^{+} .
$$

The family $F_{A T \epsilon}$ approximates the Mumford-Shah functional in the sense of $\Gamma$ convergence as you can see in $[1,7]$, since the equicoercivity properties are verified, up to subsequences, the $u_{\epsilon}$ of any global minimizer $\left(u_{\epsilon}, v_{\epsilon}\right)$ of the $F_{A T \epsilon}$ converges to a global minimizer $u$ of M-S.

The function $S_{\epsilon}$ is a sequence of edge variables that provides the approximation of $S_{u}$ while $\varepsilon \rightarrow 0^{+}$.

\section{DiscretizATION AND NUMERICAL APPROXIMATIONS}

In many applications of image processing, it is usual to manage the image domain with its pixel definition. We consider $\Omega$ as an open and limited subset of $\mathbb{R}^{2}$ (rectangular images). We define the image as the brightness-intensity function $g: \Omega \longrightarrow \mathbb{R}$ (scaled between $0, \ldots, 2^{s}-1$, for $s$ gray-tones) considered in gray scale. We adopt the elliptic systems of equations as a support theory and the mesh grid equal to the points density domain. For the X-MET 2D model the lattice is reduced, due to the cropping step at $[1005 \times 140]$ points for every 680 frame. The co-culture system is treated as a $3 \mathrm{D}$ data of $[240 \times 320 \times 458]$ points. We use a numerical scheme based on explicit finite differences, over the rectangle $\Omega$ with step $h$; this way we obtain $(x, y)=(i h, j h)$ for $0 \leqslant i, j \leqslant N$. Reducing $\Omega$ to a square of side 1 and taking $h=\frac{1}{N}$, discrete coordinates will become: $u(i h, j h) \cong u_{i, j}$ and $S(i h, j h) \cong S_{i, j}$.

\subsection{Euler equations}

The numerical scheme is composed of two coupled parts. We begin by putting $u_{0}=g$ and $S_{0}=1$. and at every step we calculate $u_{1}$ for $S_{0}=1$ solving a linear elliptic equation and find $S_{1}$ from the second equation; this process is repeated for 
a fixed number of iterations. Some scheme for the Ambrosio-Tortorelli segmentation problem can be found on Spitaleri et al. article [12].

In order to determine the minimum of the functional we adopt the continuous theory given in Birindelli and Finzi Vita [3] adapted to a finite difference mesh-grid through the following iterative scheme by giving a maximum number of iteration $N i t$ and a tolerance $\epsilon$, and then constructing:

$$
S_{0}=1, u_{0}=g
$$

$n=1,2, \ldots, N$ it find $u_{n}$, by solving:

$$
\begin{cases}\operatorname{div}\left(\left(S_{n-1}^{2}+K_{\epsilon}\right) \nabla u_{n}\right)=\mu\left(u_{n}-g\right) & \text { in } \Omega \\ \frac{\partial u_{n}}{\partial \mathbf{n}}=0 & \text { in } \partial \Omega\end{cases}
$$

and $S_{n}$ by solving:

$$
\begin{cases}\alpha \epsilon \Delta S_{n}=S_{n}\left|\nabla u_{n}\right|^{2}-\frac{\alpha}{4 \epsilon}\left(1-S_{n}\right) & \text { in } \Omega \\ \frac{\partial S_{n}}{\partial \mathbf{n}}=0 & \text { in } \partial \Omega\end{cases}
$$

stop for $n=N i t$.

For any $n>1$ there exists $u_{n}$ an $S_{n}$ solution of the respective system which satisfies the bounds:

$$
\left\|u_{n}\right\|_{L^{\infty}} \leq\|g\|_{L^{\infty}} .
$$

The above statements are the supporting theory for the continuous model and allow for the discretization even if the data is unbounded. However, in our case the given data was limited in its intensity profile.

\subsection{The discrete divergence}

In a numerical scheme a function $u \in \mathbb{R}^{2}$ can be approximated by finite difference, its first order variation on the $x$ and for $y$ direction is:

$$
\frac{\partial u}{\partial x} \cong \frac{u_{i+1, j}-u_{i, j}}{h}, \frac{\partial u}{\partial y} \cong \frac{u_{i, j+1}-u_{i, j}}{h} .
$$

The divergence of the vector valued function $Z(x, y) \nabla u(x, y)$ by second order of centered difference is given by

$$
\begin{gathered}
\operatorname{div}(Z(x, y) \nabla u(x, y)) \cong Z_{i+\frac{1}{2}, j}\left(u_{i+1, j}-u_{i, j}\right)-Z_{i-\frac{1}{2}, j}\left(u_{i, j}-u_{i-1, j}\right)+ \\
+Z_{i, j+\frac{1}{2}}\left(u_{i, j+1}-u_{i, j}\right)-Z_{i, j+\frac{1}{2}}\left(u_{i, j-1}-u_{i, j}\right)
\end{gathered}
$$

where

$$
\begin{aligned}
Z_{i+\frac{1}{2}, j} & =\frac{1}{2}\left(Z_{i+1, j}+Z_{i, j}\right) ; Z_{i-\frac{1}{2}, j}=\frac{1}{2}\left(Z_{i, j}+Z_{i,-1 j}\right), \\
Z_{i, j+\frac{1}{2}} & =\frac{1}{2}\left(Z_{i, j+1}+Z_{i, j}\right) ; Z_{i, j-\frac{1}{2}}=\frac{1}{2}\left(Z_{i, j}+Z_{i, j-1}\right) .
\end{aligned}
$$

The $n^{t h}$ approximated item of the sequence of the Ambrosio-Tortorelli functional, by fixing every direction of the space, the term $Z(x, y)=\left(S^{2}(x, y)+K_{\epsilon}\right)$ becomes for the $x$ variable:

$$
\frac{\partial}{\partial x}\left(\left(S^{2}+K_{\epsilon}\right) \frac{\partial u}{\partial x}\right) \cong
$$




$$
\frac{1}{2 h^{2}}\left[\left(S_{i+1, j}^{2}+S_{i, j}^{2}\right)\left(u_{i+1, j}-u_{i, j}\right)+\left(S_{i, j}^{2}+S_{i-1, j}^{2}\right)\left(u_{i-1, j}-u_{i, j}\right)\right],
$$

for the $y$ variable:

$$
\begin{aligned}
& \frac{\partial}{\partial y}\left(\left(S^{2}+K_{\epsilon}\right) \frac{\partial u}{\partial y}\right) \cong \\
& \frac{1}{2 h^{2}}\left[\left(S_{i, j+1}^{2}+S_{i, j}^{2}\right)\left(u_{i, j+1}-u_{i, j}\right)+\left(S_{i, j}^{2}+S_{i, j-1}^{2}\right)\left(u_{i, j-1}-u_{i, j}\right)\right] .
\end{aligned}
$$

In Image Processing the lattice has a spatial density which is equivalent to image resolution, so we use here finite differences with node resolution equal to the spatial grid-step, then the discretized equation will look as follows

$$
\begin{gathered}
\left(S_{i+1, j}^{2}+S_{i, j}^{2}\right)\left(u_{i+1, j}-u_{i, j}\right)+\left(S_{i, j}^{2}+S_{i-1, j}^{2}\right)\left(u_{i-1, j}-u_{i, j}\right)+ \\
\quad+\left(S_{i, j+1}^{2}+S_{i, j}^{2}\right)\left(u_{i, j+1}-u_{i, j}\right)+\left(S_{i, j}^{2}+S_{i, j-1}^{2}\right)\left(u_{i, j-1}-u_{i, j}\right)= \\
=\mu h^{2}\left(u_{i, j}-g_{i, j}\right) .
\end{gathered}
$$

The discrete Laplacian for a function $u$ is:

$$
\Delta u(x, y):=u_{x x}+u_{y y} \cong \frac{1}{h^{2}}\left[u_{i+1, j}+u_{i-1, j}-4 u_{i, j}+u_{i, j+1}+u_{i, j-1}\right] .
$$

Within $\Omega$, the second equation, using a five-point stencil, will be

$$
\alpha \epsilon\left(S_{i+1, j}+S_{i-1, j}+S_{i, j+1}+S_{i, j-1}-4 S_{i, j}\right)=h^{2} S_{i, j}|\nabla u|_{i, j}^{2}-h^{2} \frac{\alpha}{4 \epsilon}\left(1-S_{i, j}\right) .
$$

The problem being elliptic, we use a discretization such that we get centered finite differences; therefore for 2 points stencil: let $|\nabla u|_{i, j}^{2}:=|\widetilde{\nabla} u|_{i, j}^{2}$

$$
|\widetilde{\nabla} u|_{i, j}^{2} \cong \frac{1}{4 h^{2}}\left(\left(u_{i+1, j}-u_{i-1, j}\right)^{2}+\left(u_{i, j+1}-u_{i, j-1}\right)^{2}\right)
$$

for nodes inside $\Omega$.

Or by other formulation by 3 points stencil: let $|\nabla u|_{i, j}^{2}:=|\widetilde{\nabla} u|_{i, j}^{2}$

$$
|\widetilde{\widetilde{\nabla}} u|_{i, j}^{2} \cong \frac{\left(u_{i+1, j}-u_{i, j}\right)^{2}+\left(u_{i, j}-u_{i-1, j}\right)^{2}}{8 h^{2}}+\frac{\left(u_{i, j+1}-u_{i, j}\right)^{2}+\left(u_{i, j}-u_{i, j-1}\right)^{2}}{8 h^{2}} .
$$

Then

$$
S_{i, j}=\frac{\alpha \epsilon \widehat{S}_{i, j}+\alpha \frac{h^{2}}{4 \epsilon}}{4 \alpha \epsilon+h^{2}|\nabla u|_{i, j}^{2}+\alpha \frac{h^{2}}{4 \epsilon}} .
$$

where $\widehat{S}_{i, j}=S_{i+1, j}+S_{i-1, j}+S_{i, j+1}+S_{i, j-1}$.

The discretized $u$ becomes:

$$
\begin{gathered}
u_{i, j}=\frac{\left(S_{i, j}^{2}\right)\left(u_{i+1, j}+u_{i-1, j}+u_{i, j+1}+u_{i, j-1}\right)}{\left(\mu h^{2}+4 S_{i, j}^{2}+S_{i+1, j}^{2}+S_{i-1, j}^{2}+S_{i, j+1}^{2}+S_{i, j-1}^{2}\right)}+ \\
+\frac{\left(S_{i+1, j}^{2}\right)\left(u_{i+1, j}\right)+\left(S_{i-1, j}^{2}\right)\left(u_{i-1, j}\right)+\left(S_{i, j+1}^{2}\right)\left(u_{i, j+1}\right)+\left(S_{i, j-1}^{2}\right)\left(u_{i, j-1}\right)+\mu h^{2} g_{i, j}}{\left(\mu h^{2}+4 S_{i, j}^{2}+S_{i+1, j}^{2}+S_{i-1, j}^{2}+S_{i, j+1}^{2}+S_{i, j-1}^{2}\right)} .
\end{gathered}
$$

We extend the equations for the case of three dimensional data, such as the coculture system. We calculate the coefficients of the final formulation of the algorithm. 
3.2.1. System of equations and boundary condition. Neumann boundary condition can be introduced by dummy nodes on the image perimeter, so we obtain the null derivatives at the border; for $j=0$ and $i=1, \ldots, N$ with $j=-1$ and $y=-h$ the system:

$$
\left\{\begin{array}{l}
u_{i, 0}-u_{i,-1}=0 \\
S_{i, 0}-S_{i,-1}=0
\end{array} \quad i=1, \ldots, N-1\right.
$$

and so on for the remaining sides, see [8]. The symmetrical terms disappear by rewriting equation on the border, then we obtain the algebraic system with $2(N+1)^{2}$ unknown variables:

$$
\left\{\begin{array}{l}
A U=b \\
B S=e
\end{array} .\right.
$$

It can be solved by fixing $\mathrm{S}$ first and then $\mathrm{U}$ to get the following system: the recurrent two-step algorithm, uncouples the system at every iterative step, calculates the first equation, replaces it in the second one and then replaces the result back into the first equation.

3.2.2. Matrix row sorting. For the equations pair an algorithm is used, which enables a transformation of the associated matrix into a vector by performing a row sorting for $U \in \mathbb{R}^{(N+1)^{2}}$. Aligning rows back to back, an array:

$$
A \in \mathcal{M}\left((N+1)^{2},(N+1)^{2} ; \mathbb{R}\right),
$$

with PENTA-DIAGONAL structure, is obtained. A five-point cross scheme appears in respect to the variable $u_{i, j}$. The system can be solved by an iterative relaxation method such as Jacobi, with parallelized cycle, or Gauss-Seidel, both by Array Diagonal Dominance rule to assures the convergence [8].

3.2.3. Time dependence in $\nabla$ for $\mathbf{X}$-MET. The Gradient calculus on a Lattice of $h$ spacing is implemented by following the optical flow model (see [2, p. 184]), we introduce the time dependence in the gradient term $\left(|\nabla u|^{(f)}\right)^{2}$ respectively for two or three points stencil

$$
\begin{aligned}
& \left(|\nabla u|_{i, j}^{(f)}\right)^{2}=|\nabla u|_{i, j}^{2}+\frac{1}{4 h^{2}}\left(\left(u_{i, j}^{(f-1)}-u_{i, j}^{(f+1)}\right)^{2}\right), \\
& \left(|\nabla u|_{i, j}^{(f)}\right)^{2}=|\nabla u|_{i, j}^{2}+\frac{1}{4 h^{2}}\left(\frac{\left(u_{i, j}^{(f-1)}-u_{i, j}^{(f)}\right)^{2}+\left(u_{i, j}^{(f)}-u_{i, j}^{(f+1)}\right)^{2}}{2}\right) .
\end{aligned}
$$

It represents a new formulation of the model with a dynamic mean between frames. The square gradient is point by point calculated over function $u$ as the difference in brightness intensity between the preceding and the following frame. Globally, the variability of this term is mostly due to the strength of ROIs walls. In the presence of a continuous movement we are in a condition to assume regularity for function $g_{I}$ over time. Only a conjecture on continuous model to support the time dependence in gradient calculus was possible due to the difficulty to extend the M-S theory [8], in our case only the test on the images produces some interesting enhance results for future developments. A refined theory for a new functional formulation with time dependence in edge selection is worked on. 


\subsection{3d extension}

To achieve optimal recognition of the cells and to recognize their rhythmical pulse we regard it as solid material by imagining every frame as the slide of a 3 -d array. The theory supports $n$-dimensional results and formulation, so if we use the indices $i, j, l$ as the three dimensions, we have the formulation

$$
S_{i, j, l}=\frac{\alpha \epsilon \widehat{S}_{i, j, l}+\alpha \frac{h^{2}}{4 \epsilon}}{6 \alpha \epsilon+h^{2}\left|\nabla u_{i, j, l}\right|^{2}+\alpha \frac{h^{2}}{4 \epsilon}} .
$$

where $\widehat{S}_{i, j, l}=S_{i+1, j, l}+S_{i-1, j, l}+S_{i, j+1, l}+S_{i, j-1, l}+S_{i, j, l+1}+S_{i, j, l-1}$.

$$
\begin{aligned}
& u_{i, j, l}=\frac{\left(S_{i, j, l}^{2}\right)\left(u_{i+1, j, l}+u_{i-1, j, l}+u_{i, j+1, l}+u_{i, j-1, l}+u_{i, j, l+1}+u_{i, j, l-1}\right)}{\left(\mu h^{2}+6 S_{i, j, l}^{2}+S_{i+1, j, l}^{2}+S_{i-1, j, l}^{2}+S_{i, j+1, l}^{2}+S_{i, j-1, l}^{2}+S_{i, j, l+1}^{2}+S_{i, j, l-1}^{2}\right)}+ \\
& +\frac{\left(S_{i+1, j, l}^{2}\right)\left(u_{i+1, j, l}\right)+\left(S_{i-1, j, l}^{2}\right)\left(u_{i-1, j, l}\right)+\left(S_{i, j+1, l}^{2}\right)\left(u_{i, j+1, l}\right)+\left(S_{i, j-1, l}^{2}\right)\left(u_{i, j-1, l}\right)}{\left(\mu h^{2}+6 S_{i, j, l}^{2}+S_{i+1, j, l}^{2}+S_{i-1, j, l}^{2}+S_{i, j+1, l}^{2}+S_{i, j-1, l}^{2}+S_{i, j, l+1}^{2}+S_{i, j, l-1}^{2}\right)}+ \\
& +\frac{\left(S_{i, j, l+1}^{2}\right)\left(u_{i, j, l+1}\right)+\left(S_{i, j, l-1}^{2}\right)\left(u_{i, j, l-1}\right)+\mu h^{2} g_{i, j, l}}{\left(\mu h^{2}+6 S_{i, j, l}^{2}+S_{i+1, j, l}^{2}+S_{i-1, j, l}^{2}+S_{i, j+1, l}^{2}+S_{i, j-1, l}^{2}+S_{i, j, l+1}^{2}+S_{i, j, l-1}^{2}\right)} .
\end{aligned}
$$

\section{Applichtions AND RESUlts}

We show a formulation of the standard model problem for image segmentation by taking a new approach to pre-treated movies data in both systems. The regular-

Figure 4. X-MET real Image frame.

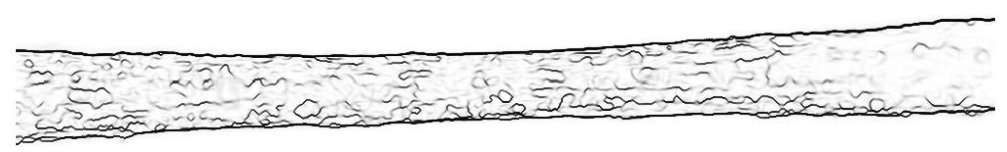

Figure 5. S: Grad time processed frame Set of edges.

ization and edge enhance obtained by pre-process methods produce homogeneous areas such as a "cartoon" image. In particular the pixels closest to the edge are emphasized over others. Then we can compute the ROIs area over the frames. The original frame of X-MET Fig. 4 as input data to the grad-time M-S produce the Fig. 5 the edge set representing the perimeter of the areas. The regularized 


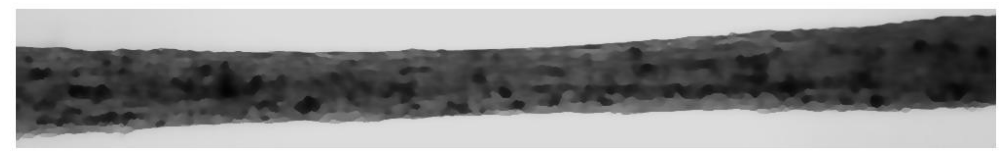

Figure 6. U: Grad time processed frame used for ROIs selection filter by MS 2D regularization:

$$
\lambda=5.0, \alpha=0.0002 \text {. }
$$

Image now presents better defined regions of iso-level intensity see Fig. 6. The follower algorithm consists of an appropriate region cropping containing the selected ROI and its possible variability. A threshold filter with lower and higher value of gray cut is used to transform images-frame in duo-tone; black and white (BW) see Fig. 7. The explained procedure was iterated for all frames. So we can map the interesting ROIs, and compute the properties such as Centroid, Areas, and so on to build the structure, see Fig. 8. So we can record the structure of properties all over the frames. Different data sets for different stimulation experiments produce a table of strength reaction useful to measure the bio-mechanical properties, which was validated by the Microforce transducer data Fig. 13.

\subsection{Protocol and results for X-MET movie processing}

The schema of the elaboration is resumed in the step necessary to evaluate the position of every ROIs. Once the first frame is used the follower algorithm builds the sequence of the structure with the measured values on all frames. See Fig. 7.

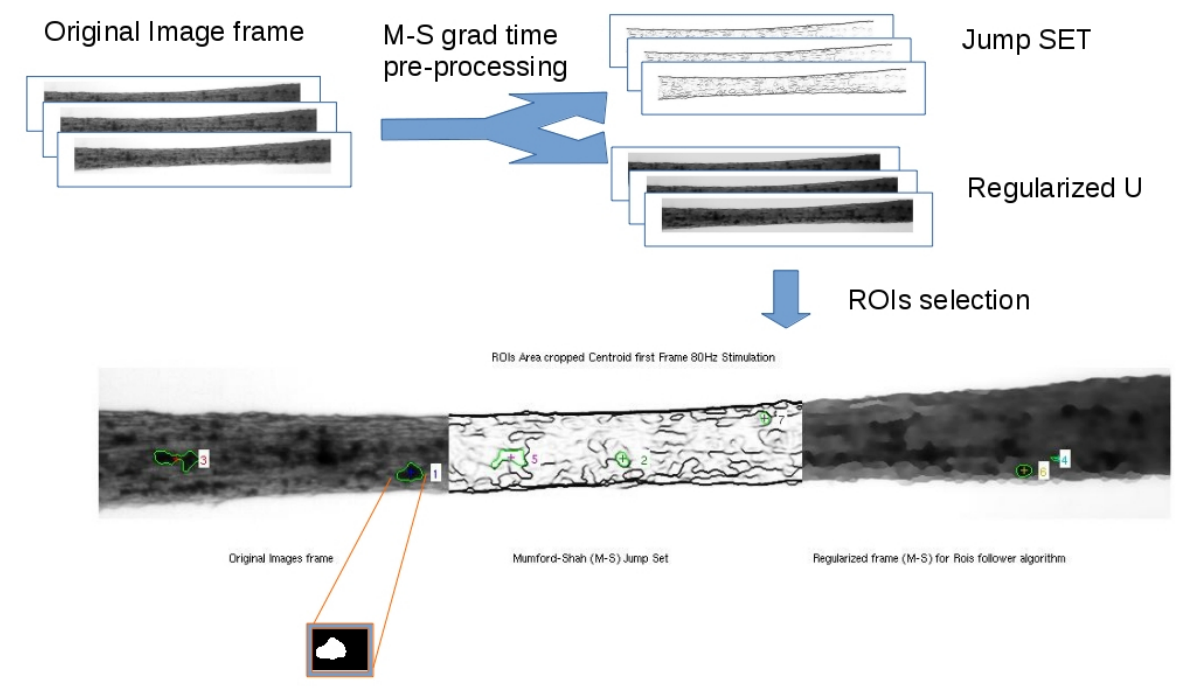

ROI 1 cropped bw

Figure 7. X-MET elaboration protocol, the multiple image represents a frame sequence in the preprocessing routine that gives back an enhanced regularized $\mathrm{U}$ and the relative $\mathrm{S}$ of jump set (the edges of recognized areas), at bottom the areas representing interesting ROIs (green color and + the centroid) the transformed ROI 1 in BW color is the final result. 
4.1.1. ROI structure parameters. Every ROI selected is mapped with the structure by the follower algorithm. So we can identify the measured values with a recorded array. The data type is generally double and the various fields that represent dynamic values present an array dimension due to the frame number of the movie as in Fig. 8. The centroid of the tracked area looks like Fig. 9.

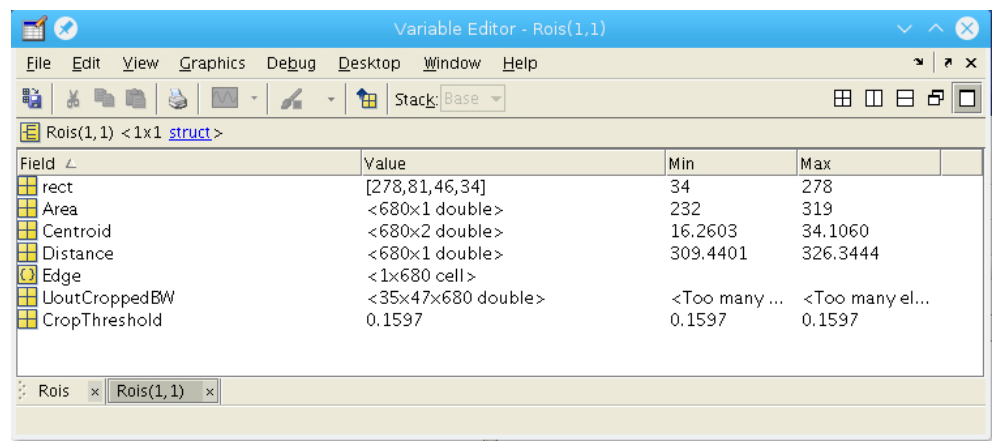

Figure 8. ROI structure of measured parameter, struct with: The rectangle of ROI movements, Array of ROI's area pixel Number x frame Number, Centroid X,Y x Frame Number, Edge Map of ROI x,y points x Frame Number, Cropped Image Black and White x Frame Number, Threshold value filter.

The X-MET setup is configured to measure at different frequency stimulation the

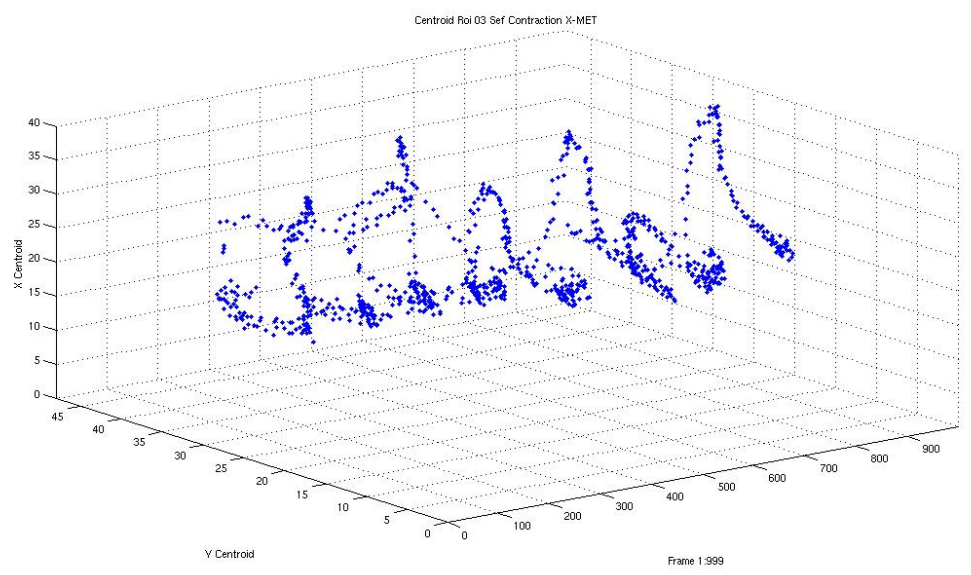

Figure 9. Centroid position over the frames of the ROI 01 cropped area in recognized movements limits, $(46 \times 34$ pixel 680 frames $)$.

strength between the fixed point and the micro force transducer. The movements, of the selected ROIs, over the $x$ axis present the typical characteristics of the muscle contraction [4]. Here we present the evaluation axis, in pixel units, of the component $\mathrm{X}$ of the Centroid for every frequency stimulation of the ROI 1 Fig. 10 and all ROIs at single frequency Fig. 12. Every stimulation frequency presents 


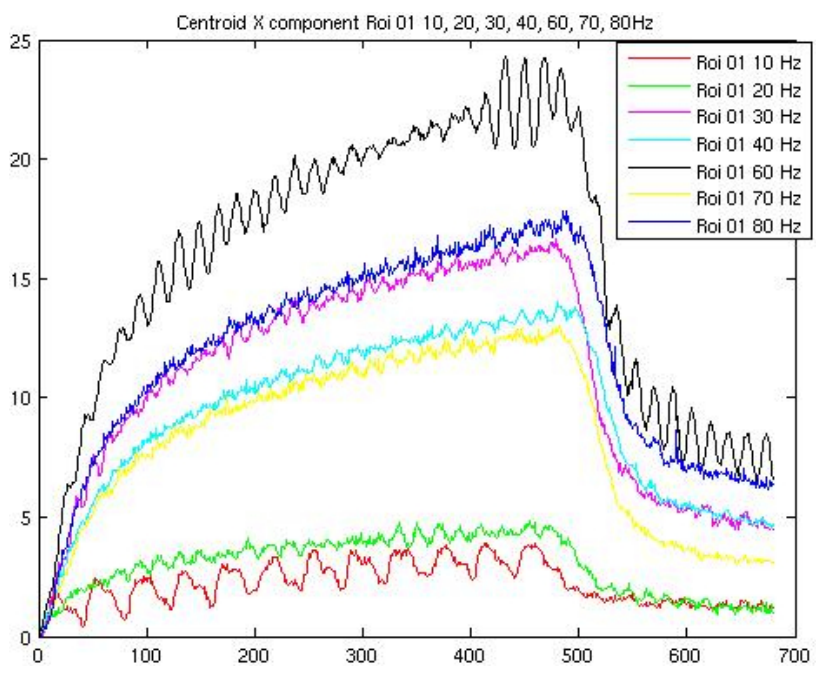

Figure 10. X-MET ROI 1 at frequency stimulation 10, 20, 30, 40,60, 70, 80 [Hz], in y axis the longitudinal value of measured movement of centroid in $\mathrm{x}$ axis the frames number.

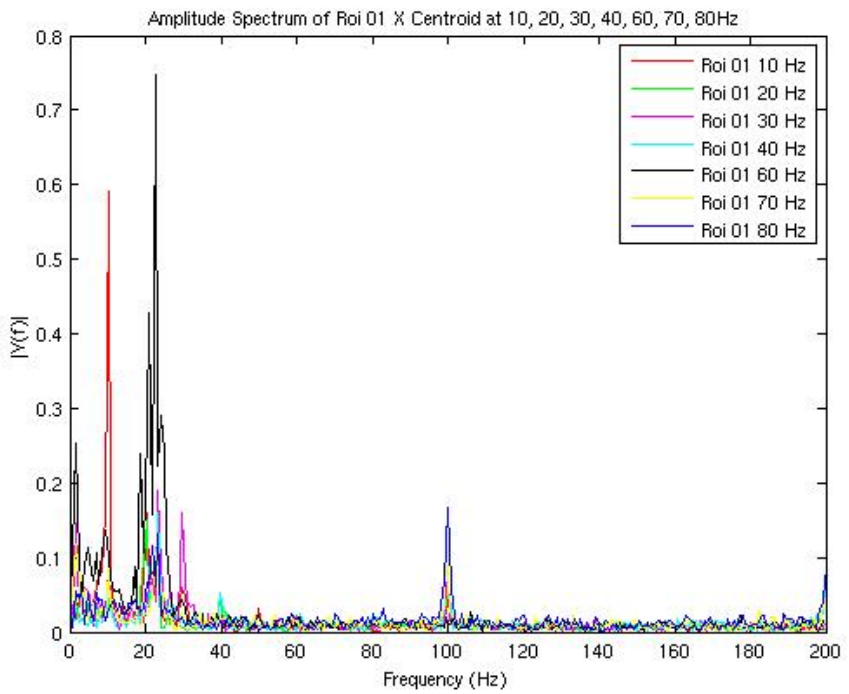

Figure 11. ROI 1 Amplitude spectrum frequency at stimulation 10, 20, 30, 40, 60, 70, $80 \mathrm{~Hz}$, by an FFT, the interpretation are in course of developments.

a different trend. For the X-MET the tetanic force increased with increasing frequency up to $70-80 \mathrm{~Hz}$ but, on average, the asymptotic value is already achieved at $60 \mathrm{~Hz}$. The errors in the follower algorithm in the Fig. 12, due to an unrecognized ROIs area, are recovered at next frame. This suggests a better performance than the previously used software for fracture recognition. Indeed X-MET's strain field 


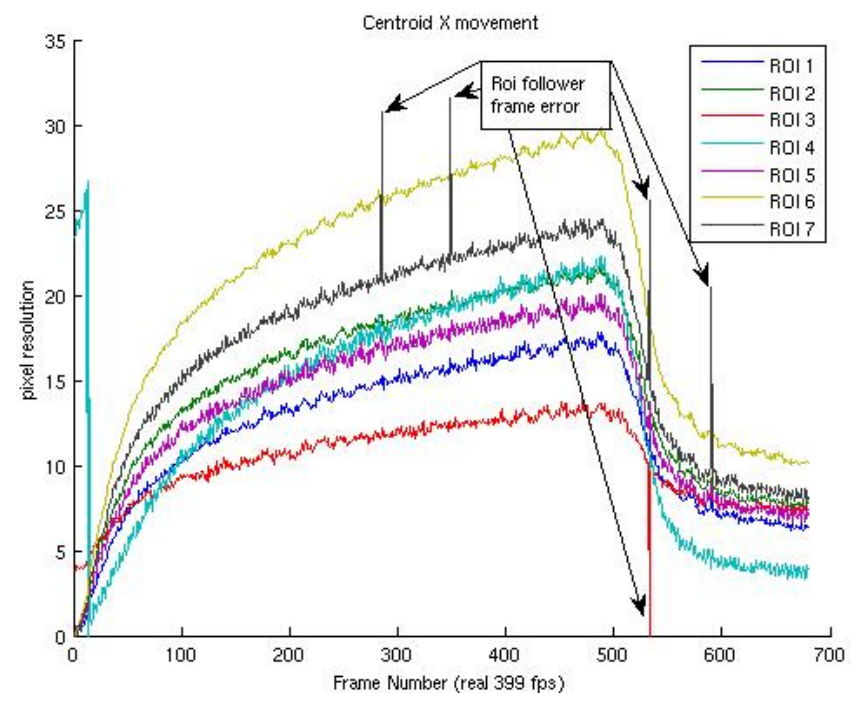

Figure 12. X-MET, ROIs Centroid X Component at $80 \mathrm{~Hz}$ stimulation in $y$ axis the longitudinal value of measured movement of centroid in $\mathrm{x}$ axis the frames number. The errors refer to an unrecognized ROI at specified frame number.

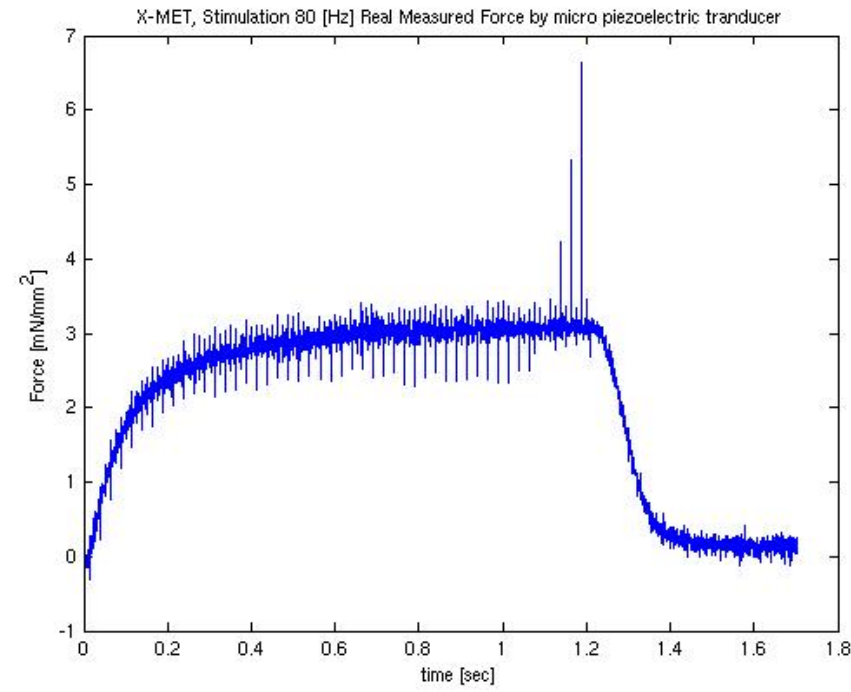

Figure 13. Example of force response obtained stimulating an X-MET by an electrical stimulation of $80 \mathrm{~Hz}$.

during electrical stimulations was previously measured through the use of a technique based on Digital Image Correlation (DIC) [10]. The correlation algorithm divides the first image into a fixed and regular grid of square elements. A similar operation is then performed on all the subsequent deformed images, arranging the 
nodes of the deformed grid. In this way, the sub-images, from the re-sampling operation, look as close as possible to the corresponding sub-images of the image used as reference. An evaluation of the standard deviation (SD) obtained when computing these average values, is a further marker of the tissues homogeneity. Muscle jerk or quick jumps cause to the follower to lost the ROI but the DIC cannot recover this error, than we have a better performance and higher definition by our methods.

\subsection{Analysis of coupled cells movements in a co-culture system.}

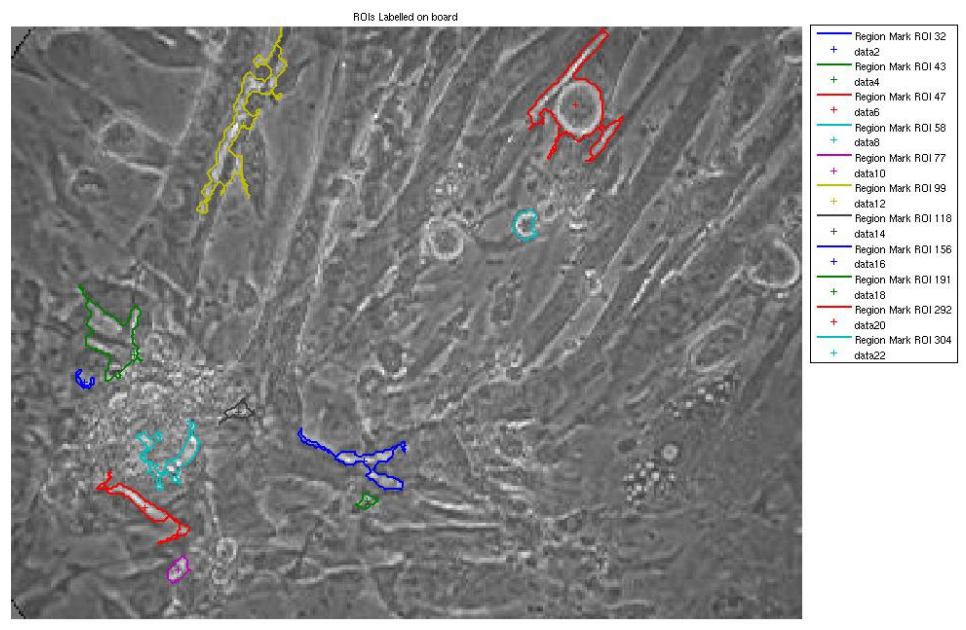

Figure 14. Interesting ROIs on co-culture board, after the pre-processing elaboration by the $3 \mathrm{D}$ algorithm, the long cell selected represents the myotubes cell's type and the circular one the cardiomyocytes. By M-S 3D $(\lambda=0.02, \alpha=0.0001)$.

We are not interested in retrieving the temporal integration but the right definition of the selected cell's type over the time. So the choice of space and time scale was set equal to the pixel and frame definition. Electrical coupling of skeletal and cardiac cells is a research field of movies analysis, and just a sketch is presented. Due to a low resolution and few frame rate movies, the protocol is only the first test to measure the correlation between the selected ROIs. In a future release of the two stages software, we hope to improve the myotubes and cardiomyocytes detection. The M-S algorithm for 3D segmentation of solid objects can be used in quality control of industrial production and other research fields. The automatic ROIs selection over the 3D data, gives us numerous different objects. The technique of $3 \mathrm{D}$ neighborhood (bwlabeln() function in Matlab ${ }^{\circledR}$, reduces the amount of possible objects (from 16642 to 1498 and 11 when we set $6,18,26$ connected-neighborhood 3D connectives after the threshold bw filtering) the best responsive values were obtained with 18 connected-neighborhood. Then we can select the ROIs representing both cell types. On these ROIs we measure the cross-correlation of their contractile response, see Fig. 14. In particular some ROIs were selected to understand the correlation between their movements the 

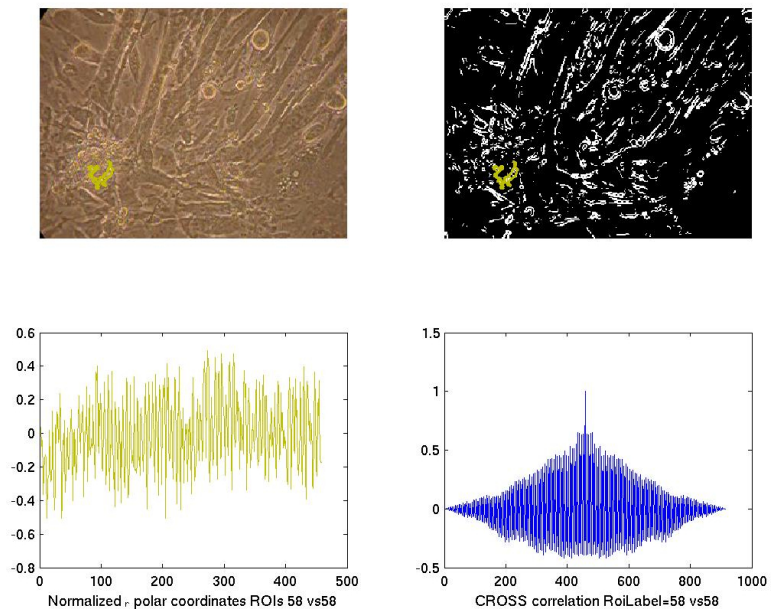

Figure 15. Cross-correlation of the same cell, the symmetric profile of the auto-correlation proves the related movements of equal cell's type.
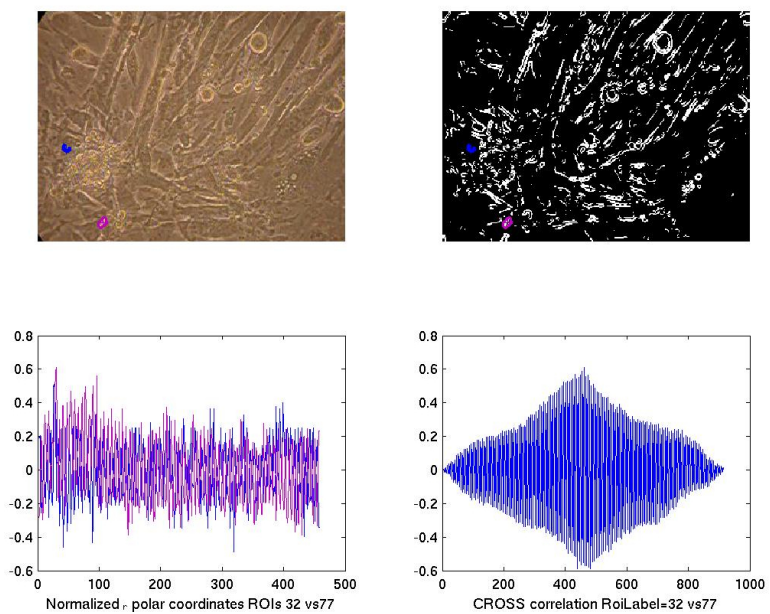

Figure 16. Similar cell's type correlation, same cell types in different location present quasisynchronized pulse.

values of interest are between cardiomyocyte (circular cell) and myotubes (long profiles cell) or between the same types in different positions. Different choices are possible to evaluate cross-correlation between significant ROIs, here we view an auto-correlation to understand and validate the results. We transform the centroid Cartesian coordinates in Polar and plot the module $\rho$. Fig. 15 and in the left bottom axis of the figures the y axis represents the normalized $\rho$ of polar coordinates of centroid's ROIs. As known by literature cardiomyocytes exhibit a synchronous beating, and so by selecting ROIs containing distant cardiomyocytes we can evaluate the real correlation. As expected the selection of two different cardyomyocites 

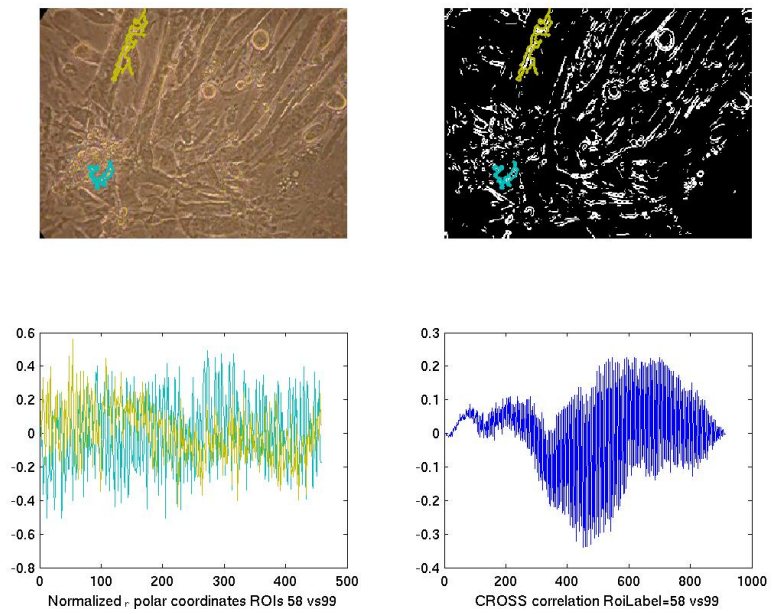

Figure 17. Different cell's type correlation, no further related pulse recognized.

show a good correlation Fig. 16. On the other hand, different cellular types Fig. 17 (myotubes in yellow and cardiomyocyte in cyan) show less correlation.

\section{Conclusion And Development}

Many trial tests are necessary to establish a better parameter choice in the functional minimization. We observe a little flash in the movies due to the digital Camera setup. Laboratory systems of illumination utilizing fluorescent lamps, hid lamps or neon tubing can be recognized by a fast-Fourier analysis of the frames background. Then a new measurement system can be implemented to reduce external light sources. The diode lamps for this kind of image acquisition has a better performance and avoid flickering at high frame rate recording. This choice should give more stability to the ROIs edge over the frame and avoid error of the follower algorithm. X-MET Possible technical refinement in analysing ROIs detection.

Apply new functional formulation to enhancing ROIs by time gradient all over the movies; test other ROIs follower and recognition focused on a little object; evaluate the strength between different ROIs; led based illumination at the Microscope sample basement; refine the protocol to retrieve 3D measure from Confocal System (the microscope lens settings to inspect the internal structure of muscle filaments) and understand amplitude of frequency spectrum analysis to evaluate stimulation and properties.

For the co-culture in-vitro systems we suggest to improve the Digital Image system to a better CCD resolution; by a higher frame rate Camera acquisition and a better recognition of cardiomyocytes and myotubes with an accurate parameter choice in two separate steps of the enhancer and follower algorithms. 


\section{REFERENCES}

[1] L. Ambrosio and V.M. Tortorelli, Approximation of functionals depending on jumps by elliptic functionals via $\gamma$-convergence, Communications on Pure and Appied Mathematics 43 (1990), 999-1036.

[2] G. Aubert and P. Kornprobst, Mathematical Problems in Image Processing, Partial Differential Equations and the Calculus of Variations, Springer-Verlag, New York, 2002.

[3] I. Birindelli and S. Finzi Vita, A class of quasi-linear elliptic systems arising in image segmentation, Nonlinear Differential Equations and Applications NoDEA 5 (1998), 445449.

[4] S. Carosio, L. Barberi et al., Generation of ex vivo-vascularized muscle engineered tissue (X-MET), Scientific Reports 3(1420) (2013), doi 10.1038/srep01420, 9 pp.

[5] M. R. Lewis, Rhythmical contraction of the skeletal muscle tissue observed in tissue cultures, American Journal of Physiology 38 (1915), 153-161.

[6] J.-M. Morel and S. Solimini, Variational Methods in Image Segmentation, Progress in Nonlinear Differential Equations and Their Applications, Birkhäuser, Basel, 1995.

[7] D. Mumford and J. Shah, Optimal approximation by piecewise smooth functions and associated variational problems, Communications on Pure and Applied Mathematics 42 (1989), 577-685.

[8] M. Pedone, Echocardiographic Image Processing, in: P. Di Giamberardino et al. (eds.), Computational Modeling of Objects Presented in Images, Fundamentals, Methods and Applications, Lecture Notes in Computational Vision and Biomechanics 15, Springer, 2014, 285-310.

[9] C. Pizza, A. Donoian et al., Shortening-deactivation and stretch-activation during spontaneous contraction and relaxation of bivalve cardiac muscles, in: Proceedings of the IEEE 32nd Annual Northeast Bioengineering Conference, Easton, PA, USA, 2006, 23-24.

[10] E. Rizzuto, S. Carosio et al., A digital image correlation based technique to control the development of a skeletal muscle engineered tissue by measuring its surface strain field, Medical Measurements and Applications (MeMeA) 2015, Turin, Italy, in: 2015 IEEE International Symposium on Medical Measurements and Applications (MeMeA) Proceedings, 2015, 314-318.

[11] E. Rizzuto, S. Carosio et al., A DIC based technique to measure the contraction of a skeletal muscle engineered tissue, Applied Bionics and Biomechanics 2016 (2016), Article ID 7465095, 7 pp.

[12] R. M. Spitaleri, R. March and D. Arena, Finite difference solution of Euler equation arising in variational image segmentation, Numerical Algorithms 21 (1999), 353-365.

Massimiliano Pedone, InfoSapienza ICT Center, Sapienza University of Rome, Italy e-mail: massimiliano.pedone@uniroma1.it, http://pedoneweb.phys.uniroma1.it/max/ Italy

Silvia Carosio, Center for Life NanoScience, IIT@Sapienza, Istituto Italiano di Tecnologia,

Giancarlo Ruocco, Center for Life NanoScience, IIT@Sapienza, Istituto Italiano di Tecnologia, Italy

Zaccaria Del Prete, Department of Mechanical and Aerospace Engineering, Sapienza University of Rome, Italy 
